\title{
Sixty inappropriate defibrillation shocks in several days due to lead displacement
}

\author{
David Korpas $\mathrm{PhD}^{1}$, Jana Haluzikova $\mathrm{PhD}^{1}$, Marek Penhaker $\mathrm{PhD}^{2}$, Martin Augustynek $\mathrm{PhD}^{2}$
}

\begin{abstract}
D Korpas, J Haluzikova, M Penhaker, M Augustynek. Sixty inappropriate defibrillation shocks in several days due to lead displacement. Curr Res Cardiol 2016;3(1):27-28.
\end{abstract}

BACKGROUND: Implantable cardioverter-defibrillators (ICDs) are active cardiac implants for immediate treatment of ventricular arrhythmias. They provide life-saving therapy, but may also deliver inappropriate therapy.

METHODS AND RESULTS: The present report describes a rare occurrence of many inappropriate ICD shocks because of lead dis-

The noise sensed by implanted leads may be of different origins, 1 coming from a physiological function of the body or from external sources. Although sophisticated discrimination algorithms exist and the use of bipolar leads is routine, concerns regarding electromagnetic interference (EMI) persist. Many cases of EMI that led to inappropriate shocks have been described in the literature $(1,2)$. The most important physiological false signals are pectoral or diaphragmatic myopotentials. Because of physical distances, pectoral myopotentials are usually present in the shock channel and never in the pace/sense channel. Diaphragmatic myopotentials are sometimes present only in the bipolar sensing channel and only exceptionally in the shock channel, but never in the bipolar atrial channel. These myopotential issues have also often been reported (3-6).

\section{CASE PRESENTATION}

A 57-year-old man who was on dialysis and had no structural heart disease, but first-degree heart block and right bundle branch block, presented with a history of sustained ventricular fibrillations (VFs). A single-chamber implantable cardioverter-defibrillator (ICD) with integrated bipolar single coil lead for the right ventricle was implanted. The ICD was programmed into two zones: $160 / \mathrm{min}$ for the ventricular tachycardia (VT) zone and 200/min for the VF zone. After the testing for sufficient intrinsic R-wave amplitude at the implant, the ventricular sensitivity was programmed to a nominal value of $0.15 \mathrm{mV}$ with automatic gain control. The programmed therapy was one burst of eight pulses, one ramp of eight pulses, then multiplied $41 \mathrm{~J}$ shocks for the VT zone and antitachycardia pacings (ATPs) up to 250/min plus multiplied $41 \mathrm{~J}$ shocks for the VF zone.

In the morning of the 104th day after implantation, the patient received the first VF therapy, ie, ATP and $41 \mathrm{~J}$ shock. During the next 13 days, the device recorded 896 episodes. The patient received 353 ATPs and 60 shocks of $41 \mathrm{~J}$ in total, all within these 13 days. The rest of the episodes were unsustained or untreated (Table 1). The trends recorded a decrease in the sensed R-wave and fluctuation in pacing impedance. The shock impedance showed no significant difference.

A typical episode stored in the device memory is shown in Figure 1. High and random frequency, low and variable amplitude, presented similarly in both the pace/sense and shock channels, are typical for placement shortly after implantation. A 57 -year-old overweight man received 353 antitachycardia pacings and 60 shocks of $41 \mathrm{~J}$ in total, all within 13 days. The device records show a decrease in the sensed R-waves and pacing impedance fluctuation.

CONCLUSION: The ventricular electrogram signals recorded and shock channels were typical for lead rubbing.

Key Words: Implantable cardioverter-defibrillator; Inappropriate shock; Noise

TABLE 1

Ventricular episode counter data

\begin{tabular}{lc}
\hline Total episodes & 896 \\
Treated & 270 \\
VF therapy & 83 \\
VT therapy & 0 \\
VT-1 therapy & 0 \\
Commanded therapy & \\
Non-treated & 0 \\
No therapy programmed & 71 \\
Nonsustained episodes & 472 \\
Other untreated episodes & \\
Ventricular therapy counters & 353 \\
ATPs delivered & 77 \\
ATPs, \% sussessful & 60 \\
Shocks delivered & 100 \\
$\quad$ First shock, \% successful & 319 \\
Shocks diverted &
\end{tabular}

Data presented as $n$ unless otherwise indicated. Not all episode details are stored in memory. ATPs Antitachycardia pacings; VF Venticular fibrillation; VT Ventricular tachycardia

so-called 'lead rubbing'. This can usually occur between the old ventricular pace/sense lead connected in the IS-1 ventricular port and the distal shock coil of the newly implanted defibrillator lead in the re-implanted single-chamber ICD system. However, this patient has only one lead and, moreover, with a DF4 connector.

\section{RESULT}

The reason for lead rubbing was displacement of the defibrillator lead into the superior vena cava. This was also confirmed by fluoroscopy. The diameter of the superior vena cava in this overweight patient was sufficient for the lead to 'kink', causing mechanical contact between the integrated sensing electrode and lead body during any patient movement. The overall number of false therapy shocks was impressive. To our knowledge, such a high number of shocks has never been reported. Surprisingly, the ICD battery was uncompromised, still predicting the full capacity of 12 years.

${ }^{1}$ Institute of Nursing, Faculty of Public Policies, Silesian University, Opava; ${ }^{2}$ Department of Cybernetics $\mathcal{E}$ Biomedical Engineering, Faculty of Electrical Engineering $\mathcal{E}$ Computer Science, Technical University Ostrava, Ostrava, Czech Republic

Correspondence: Dr David Korpas, Institute of Nursing, Faculty of Public Policies, Silesian University, Hauerova 4, 74601 Opava,

Czech Republic. Telephone 420-553-684-704, fax 420-553-684-839, e-mail david.korpas@fvp.slu.cz 


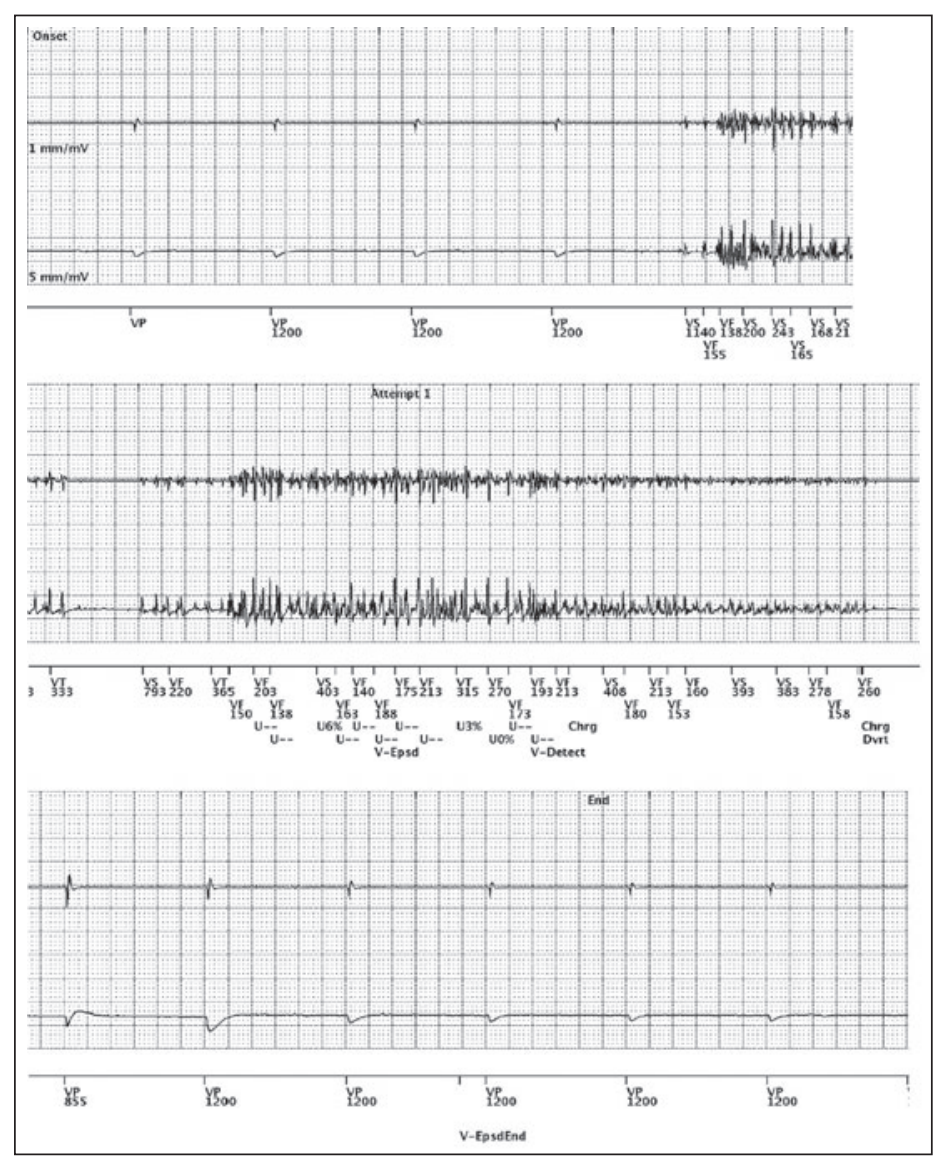

Figure 1) One of the many episodes extracted from the device memory, untreated in this case. Top panel: ventricular intracardiac electrogram used for tachyarrhythmia detection. Middle panel: shock coil electrogram (far-field). Bottom panel: marker annotations. Numbers represent appropriate cycle lengths in $\mathrm{ms}$

\section{CONCLUSIONS}

The present case emphasizes the need for proper discrimination of different types of noise sensing. EMI disturbance is often considered first; however, in reality, this is quite rare. The origin of the noise can be determined by comparing electrograms in different channels, at least right ventricle and shock change, in a single-chamber ICD.

AUTHOR CONTRIBUTIONS: All authors read the final manuscript within their respective areas of expertise and participated sufficiently in the study to take responsibility for it and accept its conclusions.

DISCLOSURES: The authors have no financial disclosures or conflicts of interest to declare.

\section{REFERENCES}

1. Kolb C, Zrenner B, Schmitt C. Incidence of electromagnetic interference in implantable cardioverter defibrillators. Pacing Clin Electrophysiol 2001;24:465-8.

2. Sabat'e X, Moure C, Nicol'as J, Sed'o M, Navarro X. Washing machine associated $50 \mathrm{~Hz}$ detected as ventricular fibrillation by an implanted cardioverter defibrillator. Pacing Clin Electrophysiol 2001;24:1281-3.

3. Barakpour H, Emkanjoo Z, Alizadeh A, Sadr-Ameli MA. Inappropriate shock delivered by implantable cardioverter defibrillator-cardiac resynchronization therapy (ICD-CRT) due to myopotential oversensing. Indian Pacing Electrophysiol J 2009;9-:71-4

4. Chen RH, Chen KP, Wang FZ, Hua W, Chen X, Zhang S. Incidence and causes of inappropriate detection and therapy by implantable defibrillators of cardioversion in patients with ventricular tachyarrhythmia. Chin Med J (Engl) 2006;119:557-63.

5. Deshmukh P, Anderson K. Myopotential sensing by a dual chamber implantable cardioverter defibrillator: Two case reports. J Cardiovasc Electrophysiol 1998;9:767-72.

6. Peters RW, Cooklin M, Brockman R, Shorofsky SR, Gold MR. Inappropriate shocks from implanted cardioverter defibrillators caused by sensing of diaphragmatic myopotentials. J Interv Card Electrophysiol 1998;2:367-70. 